\title{
Impulse Noise Reduction using Image Fusion Approach
}

\author{
Sajid Ullah Khan \\ CECOS, University, \\ Peshawar Pakistan \\ sajidullah@cecos.edu.pk
}

Wang Yin Chai

FCSIT. Universiti Malaysia Sarawak

Kuching, Malaysia

ycwang@fit.unimas.my

\author{
Najeeb Ullah Khan \\ CECOS, University, \\ Peshawar Pakistan \\ najeebullahkhan1984@gmail.com
}

\begin{abstract}
Digital image enhancement has been a hot topic during the past decades. In this paper, we have established a new fusion based approach for impulse noise reduction from multi-sensors images. Image fusion is an important technique used in remote sensing, military and medical applications. In the proposed approach, firstly, only the uncorrupted pixels from multi-sensor images are grouped and then all those grouped pixels are filter with another state of the art approach for optimal results. The proposed approach is tested with several noisy images by finding the Mean Square Error, Peak Signal to Noise Ratio and Mutual Information values between the original and restored images. The experimental results show that the proposed algorithm outclasses all methods that individually de-noised and fused images.
\end{abstract}

Keywords --- image de-noising, image fusion, impulse noise, median filter.

\section{INTRODUCTION}

Digital images are normally degraded by impulse noise. The most vital task is to attenuate this noise while keeping image details. Literature has many studies regarding the said issue. The widely used impulse noise filter is median filter [1], but it affects the edges of original images especially when the noise level is high. Different remedies of the median filter have been proposed, such as Weighted median filter [2], center weighted median filter [3]. All these filters calculate the median of each pixel nevertheless if the current pixel is affected or not. Moreover, it produces serious image blurring and can disturb noise free pixels.

Early settled switching median filters provide good results only at smaller noise density [4]. A soft-switching impulse detector at the expense of computational complexity works on highly corrupted noisy images [5]. The Progressive
Switching Median Filter (PSMF) contains several iterations bringing down the computational efficiency [6]. [7] Proposes a more efficient Switching Median Filter. (Krishnan filter) based on [8] [9], which makes possible a perfect removal of impulse noise from images corrupted with salt and pepper noise and maintains a good computational efficiency. Switching median filters with Boundary Discriminative Noise Detection performs well and can achieve pleasing result even noise density up to $90 \%$ [10]. However, it is too time-consuming to be used in real applications. Directional switching median filter detect only impulse noise corrupted pixel in same way like BDND but with a bit modification [11]. A layer discrimination using non-zero adjustment element elimination and pixel restoration wiener filter approach for poisson and impulse noise detection and restoration outclasses all the stated approaches [14]. It can detect and recover corrupted pixels in an efficient way. However, it has the limitation of window size adoption and tiny character adjustment.

The idea presented in [10] [11] and [14] perform well only for single image. However, real word applications like military operation, radar images, medical application and remote sensing where we have multiple images of the same scene can differently noised due to sensors features, conversion and objects shape and proximity. The characteristic of impulse noise is arbitrarily disseminated over the whole image make the position of the same affected pixels different in each image. It is because of the uncertainty of the impulse noise. Therefore, we need syndicate the degraded pixels of all the available images into one separate image to enhance the quality and to reduce the noise absolutely. Image fusion is the process of combining multiples images in to a single image which holds the meaningful information from all images [12] [13]. In this paper, we have established 